\title{
Anterior Column Realignment (ACR) With and Without Pre- ACR Posterior Release for Fixed Sagittal Deformity
}

\author{
JEFFREY M. HILLS, MD, ${ }^{1}$ S. TIM YOON, MD, PHD ${ }^{2}$ JOHN M. RHEE, MD, ${ }^{2}$ DHEERA \\ ANANTHAKRISHNAN, MD, MSE, ${ }^{2}$ ELLIOT KIM, MD ${ }^{2}$ KEITH W. MICHAEL, MD ${ }^{2}$ BYRON STEPHENS, \\ $\mathrm{MD}^{1}$ \\ ${ }^{1}$ Department of Orthopaedics and Rehabilitation, Vanderbilt University Medical Center, Nashville, Tennessee, ${ }^{2}$ Emory Orthopaedics and Spine Center, Atlanta, \\ Georgia
}

\begin{abstract}
Background: Multiple studies have demonstrated a strong correlation between sagittal malalignment and healthrelated quality of life measures. Thus, correction of sagittal vertical axis (SVA), pelvic tilt (PT), lumbar lordosis-pelvic incidence (LL-PI), and T1 spinopelvic inclination (T1SPi) have become a primary objective of adult spinal deformity surgery. Anterior column realignment (ACR) has emerged as a less invasive technique and while the addition of posterior osteotomies has shown greater correction in ACR, it is unknown if a pre-ACR posterior release is necessary for optimal correction. The purpose of this study was to determine if pre-ACR posterior release allows for greater sagittal deformity correction.
\end{abstract}

Methods: Seventeen patients were identified that underwent minimum 1-level ACR. Ten patients underwent an anterior-posterior surgical sequence without a pre-ACR posterior release, and 7 underwent a posterior-anteriorposterior (PAP) sequence with a pre-ACR posterior release. Radiographic outcomes at final follow-up and complications were compared.

Results: Both groups saw significant improvements in LL, LL-PI, PT, SVA, and T1SPi but the correction was not significantly different between cohorts. With the exception of PT in the PAP group, the improvements in LL-PI, PT, and SVA correlated to improvement in Scoliosis Research Society-Schwab classification. The correction achieved at the ACR level, represented by motion segment angle, was greater in the PAP group by a degree that approached statistical significance. Five patients $(29 \%)$ had 6 complications.

Conclusions: Both techniques achieved meaningful improvements in overall sagittal alignment. Our results suggest that a pre-ACR posterior release may allow for greater correction specifically at the ACR level but may not always be necessary to achieve clinically meaningful correction of sagittal plane deformity.

Level of Evidence: 3

Clinical Relevance: We present our experience with and without pre-ACR posterior release. To our knowledge, this is the first study to show that pre-ACR posterior release may achieve greater correction at the ACR level.

Other \& Special Categories

Keywords: spine, deformity, anterior column realignment, sagittal malalignment, sagittal correction

\section{INTRODUCTION}

The prevalence of adult spinal deformity has been estimated as high as $68 \%$ in adults over 60 in the United States. ${ }^{1}$ Historically, the diagnosis of spinal deformity focused on coronal plane measurements (ie, Cobb angle); however, multiple studies have demonstrated a much stronger correlation between sagittal malalignment and health-related quality of life (HRQOL) measures. ${ }^{2-4}$ Sagittal malalignment may result from prior surgery, trauma, metabolic bone disease, or multilevel degenerative disc disease. ${ }^{5}$ With a demographic shift toward an older population and an increasing rate of spinal fusions, incidence of sagittal imbalance is expected to increase. $^{5}$

Correction of sagittal deformity to an ageappropriate alignment is a primary goal of adult spinal deformity surgery. Traditionally, severe deformities were corrected with open, 3-column osteotomies, such as the pedicle subtraction osteotomy and vertebral column resections. While these procedures have sustained long-term improvements in HRQOL measures, ${ }^{6}$ they carry high morbidity with complication rates as high as $70 \%$ for revision and $46 \%$ for primary procedures. ${ }^{7}$ Anterior column realignment (ACR) has emerged as a less invasive technique utilizing a transpsoas interbody approach 


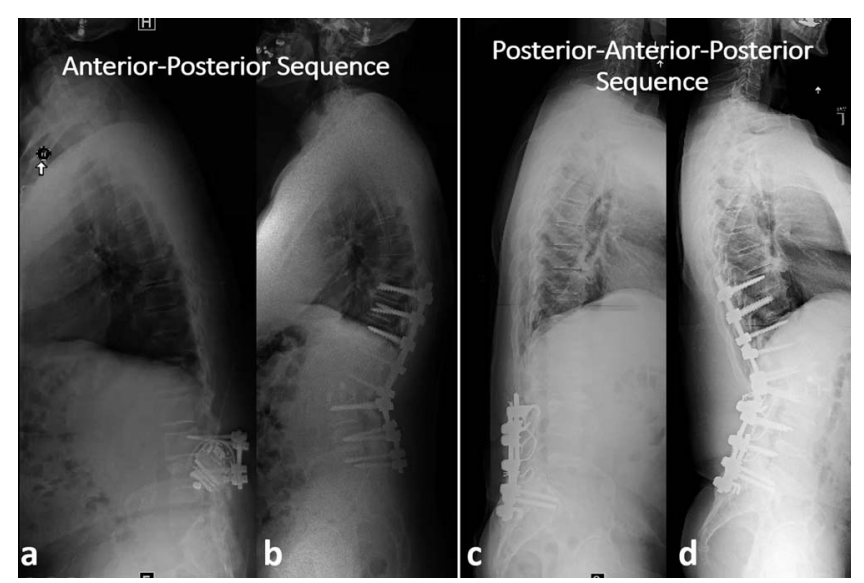

Figure 1. Representative radiographs demonstrating (a) preoperative lateral radiograph and (b) postoperative lateral radiograph following an anteriorposterior sequence for anterior column realignment; and (c) preoperative lateral radiograph and (d) postoperative lateral radiograph following a posterioranterior-posterior sequence.

with anterior longitudinal ligament release and placement of a hyperlordotic cage. While limited, studies thus far show promise for ACR to correct sagittal malalignment with similar or lower complication rates than traditional 3-column osteotomies. ${ }^{8}$ Greater deformity correction has been achieved with the addition of posterior-based osteotomies. ${ }^{9}$ However, it is unclear when, if ever, a pre-ACR posterior osteotomy is necessary for optimal deformity correction.

The purpose of this study is to determine if a preACR posterior release via a posterior-anteriorposterior (PAP) surgical sequence allows for greater sagittal deformity correction than a procedure done without a pre-ACR posterior osteotomy through an anterior-posterior (AP) surgical sequence. We aim to add to the growing body of literature on ACR outcomes to guide future prospective studies addressing this challenging problem.

\section{METHODS}

\section{Patient Cohort}

Following Institutional Review Board approval, the charts of 17 patients that underwent ACR surgery at a major academic center between August 2012 and August 2015 were retrospectively reviewed. All patients had a fixed deformity as described by Bridwell et al, ${ }^{10}$ were treated with a minimum 1-level ACR, and were over the age of 18 years. Patients underwent either an AP or PAP surgical sequence. Decision for PAP surgical sequence was surgeon preference, based on lack of motion at the ACR level on preoperative imaging.

\section{Data Collection and Outcome Measures}

Patient demographics, surgical details, and postoperative complications were collected retrospectively. Radiographs were obtained preoperatively, postoperatively, and at final follow-up (Figure 1) and reviewed to measure lumbar lordosis (LL), pelvic incidence-lumbar lordosis (PI-LL), pelvic tilt (PT), sagittal vertical axis (SVA), and T1 spinopelvic inclination (T1SPi), as previously described. ${ }^{4,11}$ Motion segment angle (MSA) was calculated by measuring the angle between the superior endplate above the ACR and the inferior endplate below the ACR.

\section{Statistical Analysis}

Mean and standard deviation for continuous variables, and frequency with ranges were used. Patient demographics, radiographic follow-up, operative details, and radiographic changes were compared between patients undergoing AP and PAP sequences. Continuous variables were compared using Mann-Whitney test. The statistical significance level was set at $P<.05$. All analyses were performed using GraphPad Prism version 6 (La Jolla, California).

\section{RESULTS}

Seventeen patients that underwent an ACR were identified. Ten patients underwent an AP sequence without a pre-ACR posterior release, and 7 patients underwent a PAP sequence, with a pre-ACR posterior release.

\section{Patient Characteristics}

There was no statistically significant difference in age, body mass index, or sex between the 2 groups. In the AP and PAP groups respectively, mean age was 66.4 and 64.3 and mean body mass index was 29.8 and 30.3. In the AP group, 6 of 10 were male $(60 \%)$, and 5 of $7(71 \%)$ were male in the PAP group (Table 1).

\section{Operative Details}

Mean radiographic follow-up was $11.75 \pm 5.5$ months in the AP group, and $14.7 \pm 11.2$ in the PAP group $(P=.79)$ (Table 2). Prior number of levels fused, final number of levels fused, number of posterior column osteotomies and number of patients with ACR-level posterior column osteoto- 
Table 1. Patient characteristics.

\begin{tabular}{|c|c|c|c|c|}
\hline Patient Characteristics & Total $(n=17)$ & AP Sequence $(n=10)$ & PAP Sequence $(n=7)$ & $P$ Value \\
\hline $\mathrm{Age}^{\mathrm{a}}, \mathrm{y} \pm \mathrm{SD}$ & $65.5 \pm 7.5$ & $66.4 \pm 8.8$ & $64.3 \pm 5.6$ & .458 \\
\hline Body mass index ${ }^{1}$ & $30 \pm 4.7$ & $29.8 \pm 4.7$ & $30.3 \pm 5$ & .981 \\
\hline Sex: male, n (\%) & $11(65 \%)$ & $6(60 \%)$ & $5(71 \%)$ & 1.0 \\
\hline
\end{tabular}

Abbreviations: AP indicates anterior-posterior; PAP, posterior-anterior-posterior.

${ }^{a}$ Values are mean $\pm \mathrm{SD}$.

mies was similar between the 2 groups. Estimated blood loss was higher in the PAP but the difference was not significant. The ACR procedure was performed on 15 levels in the AP group (mean 1.5 levels per patient, range 1-3) ranging from T12-L1 to L4-5. Each patient in the PAP group had the ACR procedure on 1 level, with levels ranging from L1-2 to L3-4. A total of 14 lateral lumbar interbody fusion procedures were performed in the AP group (1.4 per patient, range $1-3$ ), and 2 in the PAP group (0.3 per patient, range $0-1)$. Two anterior lumbar interbody fusion procedures were performed in the AP group and 5 in the PAP group. The frequency of interbody cages used in the AP group were $10^{\circ} \times 1$, $12^{\circ} \times 1,20^{\circ} \times 5$, and $30^{\circ} \times 6$, with posterior heights ranging from 6 to $10 \mathrm{~mm}$. Frequency of interbody cages used in the PAP group were: $12^{\circ} \times 1$ and $30^{\circ} \times 8$, with posterior heights ranging from 6 to 8 $\mathrm{mm}$.

\section{Complications and Fusion Status}

Of 17 patients, $5(29 \%)$ had 6 complications. Proximal junctional kyphosis occurred in 2 patients in in the AP group and 2 in the PAP group. There was 1 superficial wound dehiscence and 1 surgical infection in the PAP group. At the time of review, 8 patients from the AP group had computed tomography (CT)-confirmed fusion, the other 2 had yet to have a CT scan performed. Five of the 7 PAP patients had a CT scan completed and 4 had achieved a solid arthrodesis.

\section{Radiographic Results}

At final follow-up, LL increased from a mean $21.3^{\circ}$ to $44^{\circ}$ in the AP group $(P=.012)$, and from $27.1^{\circ}$ to $47.7^{\circ}$ in the PAP group $(P=.038)$; PI-LL improved from $36.3^{\circ}$ to $13.6^{\circ}$ in the AP group $(P=.02)$, and from $28.3^{\circ}$ to $7.8^{\circ}$ in the PAP group $(P=.023)$ (Table 3$)$. These changes correlated to an improvement in Scoliosis Research Society (SRS)-

Table 2. Operative details.

\begin{tabular}{|c|c|c|c|c|}
\hline Surgical Details & Total $(\mathbf{n}=17)^{\mathbf{a}}$ & AP Sequence $(\mathbf{n}=10)$ & PAP Sequence $(n=7)$ & $P$ Value $^{\mathrm{b}}$ \\
\hline Radiographic follow-up, mo & $9.8 \pm 5.3$ & $11.75 \pm 5.5$ & $14.7 \pm 11.2$ & .79 \\
\hline Prior number of levels fused & $3.8 \pm 1.4$ & $3.6 \pm 1.8$ & $4.1 \pm 0.7$ & .356 \\
\hline Final number levels fused & $8.1 \pm 2.9$ & $8.2 \pm 3.2$ & $7.9 \pm 2.6$ & .965 \\
\hline Estimated blood loss, $\mathrm{mL}$ & $1578 \pm 613$ & $1443 \pm 684$ & $1771 \pm 478$ & .260 \\
\hline ACR levels per patient, mean (range) & $1.3(1-3)$ & $1.5(1-3)$ & $1(1)$ & \\
\hline \multicolumn{5}{|l|}{ Levels of ACR, $\mathrm{n}$} \\
\hline T12-L1 & 2 & 2 & 0 & \\
\hline L1-2 & 6 & 2 & 4 & \\
\hline L2-3 & 6 & 4 & 2 & \\
\hline L3-4 & 7 & 6 & 1 & \\
\hline L4-5 & 1 & 1 & 0 & \\
\hline \multicolumn{5}{|l|}{ Interbody cage details, $\mathrm{n}$ (mean posterior height [range, $\mathrm{mm}]$ ) } \\
\hline 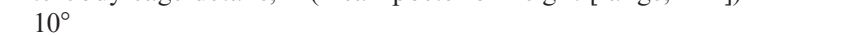 & & $1(10)$ & & \\
\hline $12^{\circ}$ & & $1(10)$ & $1(7)$ & \\
\hline $20^{\circ}$ & & $5(8.4[6-10])$ & & \\
\hline $30^{\circ}$ & & $6(7[6-10])$ & $8(6.5[6-8])$ & \\
\hline Posterior osteotomies per patient, mean (range) & $1.4(0-3)$ & $1.2(0-3)$ & $1.6(0-3)$ & \\
\hline Patients with posterior osteotomy at same level of ACR, n (\% total) & $13(76)$ & $8(80)$ & $5(71)$ & \\
\hline LLIF levels per patient, mean (range) & $0.9(1-3)$ & $1.4(1-3)$ & $0.3(0-1)$ & \\
\hline ALIF levels per patient, mean (range) & $0.4(0-2)$ & $0.2(0-1)$ & $0.7(0-2)$ & \\
\hline Complications, total & 6 & 2 & 4 & \\
\hline PJK & 4 & 2 & 2 & \\
\hline Surgical site infection & 1 & $\ldots$ & 1 & \\
\hline Superficial wound dehiscence & 1 & $\ldots$ & 1 & \\
\hline
\end{tabular}

Abbreviations: AP indicates anterior-posterior, PAP, posterior-anterior-posterior; ACR, anterior column realignment; LLIF, lateral lumbar interbody fusion; ALIF, anterior lumbar interbody fusion; PJK, proximal junctional kyphosis.

${ }^{\mathrm{a}}$ Values reported as mean $\pm \mathrm{SD}$ or mean (range).

${ }^{\mathrm{b}} P$ value is obtained from Mann-Whitney test comparing the AP sequence cohort to the PAP sequence cohort. 
Hills et al.

Table 3. Sagittal radiographic and Scoliosis Research Society-Schwab classification improvements immediately postop and at final follow-up.

\begin{tabular}{|c|c|c|c|c|c|}
\hline Radiographic Parameter & Preoperative $^{a}$ & Postoperative & Final Follow-Up & $P$ Value $^{\mathrm{b}}$ & $P$ Value $^{\mathrm{c}}$ \\
\hline \multicolumn{6}{|l|}{$\operatorname{LL}\left({ }^{\circ}\right)$} \\
\hline Total $(\mathrm{n}=17)$ & $23.7 \pm 18.2$ & $45.6 \pm 12.8$ & $45.5 \pm 12.3$ & $<.001$ & $<.001$ \\
\hline AP sequence $(n=10)$ & $21.3 \pm 20.1$ & $42.5 \pm 13.5$ & $44 \pm 13.8$ & .01 & .012 \\
\hline PAP sequence $(n=7)$ & $27.1 \pm 16$ & $50 \pm 11.2$ & $47.7 \pm 10.6$ & .023 & .038 \\
\hline \multicolumn{6}{|l|}{ PI-LL $\left({ }^{\circ}\right)^{3}$} \\
\hline Total & $33 \pm 16.4 /++$ & $11.1 \pm 11.8 /+$ & $11.2 \pm 15.5 /+$ & $<.001$ & $<.001$ \\
\hline AP sequence & $36.3 \pm 20.5 /++$ & $15.1 \pm 12.5 /+$ & $13.6 \pm 16.7 /+$ & .017 & .02 \\
\hline PAP sequence & $28.3 \pm 6.9 /++$ & $5.4 \pm 8.7 / 0$ & $7.8 \pm 14.1 / 0$ & .001 & .023 \\
\hline \multicolumn{6}{|l|}{ PT $\left({ }^{\circ}\right)^{3}$} \\
\hline Total & $25.6 \pm 9 /+$ & $15.8 \pm 9.1 / 0$ & $18 \pm 11 / 0$ & .005 & .037 \\
\hline AP sequence & $24.5 \pm 8.9 /+$ & $16.6 \pm 9.5 / 0$ & $18 \pm 11.8 / 0$ & .10 & .159 \\
\hline PAP sequence & $27.1 \pm 9.6 /+$ & $14.3 \pm 9.2 / 0$ & $18 \pm 10.7 / 0$ & .046 & .14 \\
\hline \multicolumn{6}{|l|}{ SVA $(\mathrm{cm})^{\mathrm{d}}$} \\
\hline Total & $12.3 \pm 5.2 /++$ & $4.1 \pm 3.2 /+$ & $4 \pm 3.9 /+$ & $<.001$ & $<.001$ \\
\hline AP sequence & $11.7 \pm 5.06 /++$ & $5.16 \pm 2.75 /+$ & $4.8 \pm 3.74 /+$ & .006 & .005 \\
\hline PAP sequence & $13.1 \pm 5.8 /++$ & $2.40 \pm 3.519 / 0$ & $2.4 \pm 4.1 / 0$ & .005 & .004 \\
\hline \multicolumn{6}{|l|}{ T1SPi $\left(^{\circ}\right)$} \\
\hline Total & $5.5 \pm 4.7$ & $-0.5 \pm 4.2$ & $-2.4 \pm 4.3$ & .003 & $<.001$ \\
\hline AP sequence & $5 \pm 5.8$ & $0.13 \pm 4.3$ & $-1.7 \pm 4.7$ & .07 & .024 \\
\hline PAP sequence & $6.1 \pm 3.0$ & $-1.6 \pm 4.4$ & $-3.8 \pm 3.3$ & .025 & .003 \\
\hline \multicolumn{6}{|l|}{ MSA at ACR level ( $\left.{ }^{\circ}\right)$} \\
\hline Total & $4.3 \pm 14.1$ & $20.8 \pm 10.8$ & $24.3 \pm 12.1$ & $<.001$ & $<.001$ \\
\hline AP sequence & $8.3 \pm 11.8$ & $20.9 \pm 10.1$ & $25.6 \pm 12.8$ & .006 & .002 \\
\hline PAP sequence & $-3.6 \pm 15.9$ & $20.6 \pm 12.8$ & $21.7 \pm 11.1$ & .012 & .004 \\
\hline
\end{tabular}

Abbreviations: ACR indicates anterior column realignment; AP, anterior-posterior, PAP, posterior-anterior-posterior; LL, lumbar lordosis; LL-PI, lumbar lordosis pelvic incidence; PT, pelvic tilt; SVA, sagittal vertical axis; T1SPi, T1 spinopelvic inclination; MSA, motion segment angle; SRS, Scoliosis Research Society.

${ }^{\mathrm{a}}$ Values reported as mean $\pm \mathrm{SD}$.

${ }^{\mathrm{b}} P$ value is obtained from Mann-Whitney test comparing preoperative to initial postoperative parameters.

${ }^{\mathrm{c}} P$ value is obtained from Mann-Whitney test comparing preoperative to final follow-up parameters.

${ }^{\mathrm{d}} \mathrm{SRS}-\mathrm{Sch} w a b$ classification where PI-LL of $10^{\circ}=0,10^{\circ}-20^{\circ}=+$, and $>20^{\circ}=++$; SVA: $<4 \mathrm{~cm}=0,4-9.5 \mathrm{~cm}=+,>9.5 \mathrm{~cm}=++$; PT $<20^{\circ}=0,20^{\circ}-30^{\circ}=+$,

$>30^{\circ}=+$.

Schwab Classification by 1 grade in the AP group and 2 grades in the PAP group. ${ }^{12}$ In the AP group, PT decreased corresponding to an improvement in SRS-Schwab grade that was sustained at final follow-up $\left(24.5^{\circ}\right.$ to $\left.18^{\circ}, P=.159\right)$. SVA decreased from $11.7 \mathrm{~cm}$ to $4.8 \mathrm{~cm}$ in the AP group $(P=.005)$, and from $13.1 \mathrm{~cm}$ to $2.4 \mathrm{~cm}$ in the PAP group $(P=.004)$, which corresponded to 1 SRS-Schwab grade improvement in the AP group and 2 grades in the PAP group. T1SPi improved from $5^{\circ}$ to $-1.7^{\circ}$ in the AP group $(P=.024)$, and from $6.1^{\circ}$ to $-3.8^{\circ}$ in the PAP group $(P=.003)$. MSA at the ACR level increased from a mean of $8.3^{\circ}$ to $25.6^{\circ}$ in the AP group $(P=.002)$, and from a of mean $-3.6^{\circ}$ to $21.7^{\circ}$ in the PAP group $(P=.004)$.

None of the radiographic improvements were statistically different when comparing the AP group to the PAP group (Figure 2). At final follow-up, LL increased $22.7^{\circ}$ in the AP group and $20.5^{\circ}$ in the PAP group, PI-LL decreased a by mean $22.7^{\circ}$ in the AP group and $20.5^{\circ}$ in the PAP group, PT decreased $6.5^{\circ}$ in the AP group and $9.1^{\circ}$ in the PAP group, SVA decreased by $6.9 \mathrm{~cm}$ in the AP group and 7.8 $\mathrm{cm}$ in the PAP group, and T1SPi decreased by $6.7^{\circ}$ in the AP group and $8.6^{\circ}$ in the PAP group. The mean MSA correction at the level of ACR was greater in the PAP group by a value that approached but did not reach statistical significance. The MSA increased by $13.9^{\circ}$ in the AP group compared to $24.1^{\circ}$ in the PAP group initially $(P=.152)$ and at final follow-up, the increase was $17.3^{\circ}$ in the AP group and $25.3^{\circ}$ in the PAP group $(P=.09)$.

\section{DISCUSSION}

We present our experience with 17 patients that underwent ACR for fixed sagittal imbalance. Seven patients underwent a pre-ACR posterior release (PAP group) and 10 patients did not have a preACR posterior release (AP group). Both groups saw significant improvements in LL, LL-PI, PT, SVA, and T1SPi but the correction was not significantly different between the cohorts. With the exception of PT in the PAP group, the changes in LL-PI, PT, and SVA all improved by a clinically meaningful degree at final follow-up. The correction achieved at the ACR level, represented by the mean MSA, was greater in the PAP group by a degree that approached, but did not reach statistical significance. A total of 5 patients $(29 \%)$ had 6 complications. This analysis highlights the ability of the ACR 


\section{Change in Radiographic Parameters from Pre-op to Post-op, and Pre-op to Final Follow-up}
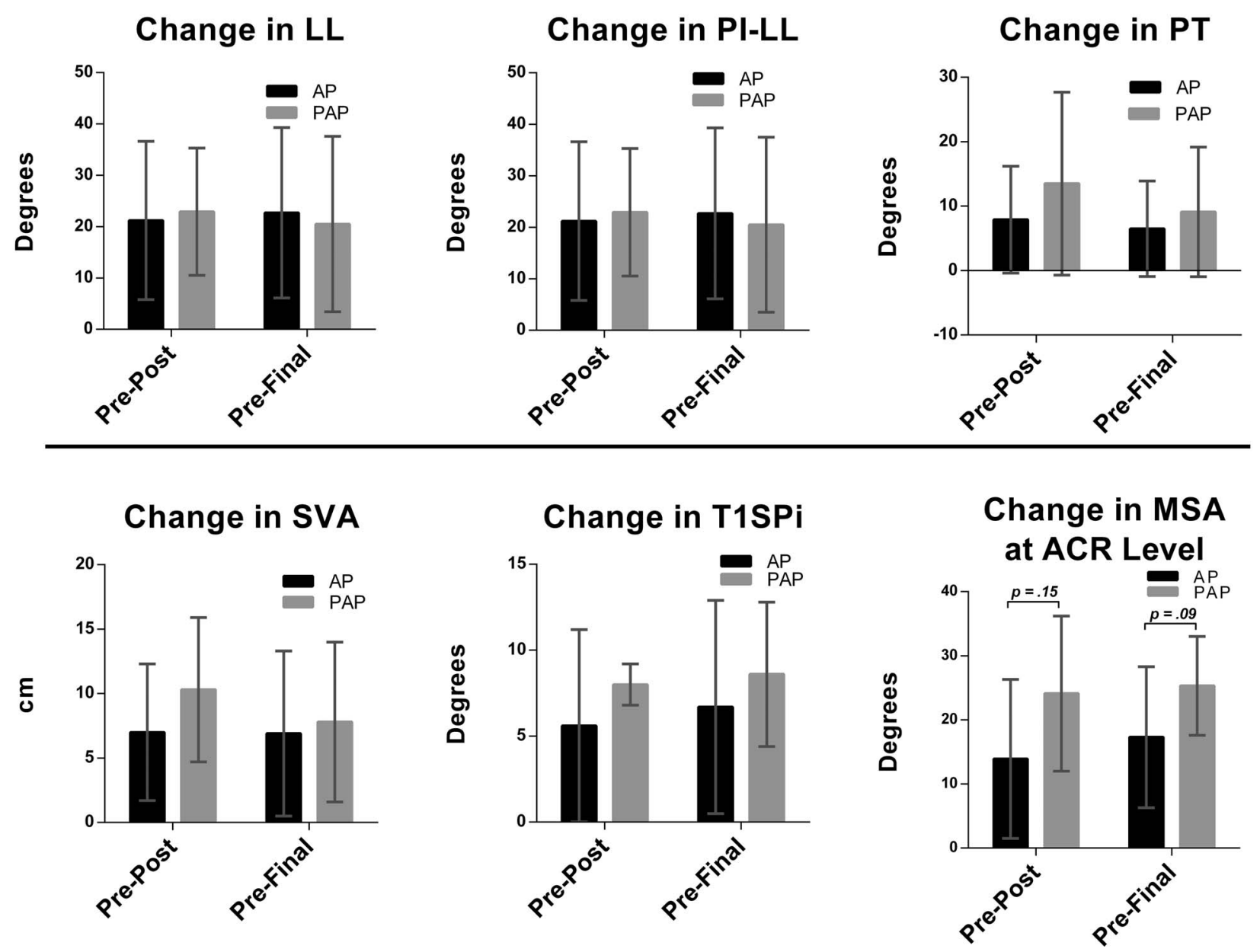

Figure 2. Change in radiographic parameters compared between anterior-posterior (AP) and posterior-anterior-posterior (PAP) cohorts. Values represent mean and error bars represent SD. The change at each time point was compared between the AP and PAP cohorts by Mann-Whitney test, and while the differences observed in motion segment angle (MSA) approached significance, none of the differences were statistically significant with a $P$ value set at .05. Abbreviations: LL, lumbar lordosis; PI-LL, pelvic incidence-lumbar lordosis; PT, pelvic tilt; SVA, sagittal vertical axis; T1-SPi, T1 spinopelvic inclination; ACR, anterior column realignment.

procedure to correct fixed sagittal imbalance with and without pre-ACR posterior release.

Sagittal plane malalignment is an increasingly recognized source of pain and has been associated with poor quality of life. ${ }^{3,13}$ Schwab et al ${ }^{11}$ demonstrated improvement thresholds in sagittal modifiers that were associated with improved HRQOL measures (LL-PI: $<10^{\circ}, 10^{\circ}-20^{\circ},>20^{\circ}$; SVA: $<4 \mathrm{~cm}, 4-9.5 \mathrm{~cm},>9.5 \mathrm{~cm}$; PT thresholds: $\left.<20^{\circ}, 20^{\circ}-30^{\circ},>30^{\circ}\right){ }^{14}$ In a prospective study, Lafage et $\mathrm{al}^{15}$ found PT and SVA correlated with HRQOL measures, but found the most significant correlations with T1SPi. ${ }^{4}$ Thus, correction of SVA, PT, LL-PI, and T1SPi have become primary objectives of adult spinal deformity surgery.,16 Traditional methods for sagittal plane deformity correction have been posterior column osteotomies for mild deformities and 3-column osteotomies such as pedicle subtraction osteotomies and vertebral column resections for severe deformities. However, these procedures carry high morbidity with complication rates up to $70 \%$ in revision surgery. ${ }^{7}$ ACR has emerged as a less invasive technique to treat sagittal imbalance with the goal of minimizing morbidity associated with deformity surgery. However, the literature on ACR is sparse. Murray et $\mathrm{al}^{17}$ reviewed 31 patients receiving 47 ACR procedures and reported an improved mean LL-PI from $26.5^{\circ}$ to $11^{\circ}$, mean LL from $32.3^{\circ}$ to $49.9^{\circ}$, and SVA from $10 \mathrm{~cm}$ to $6.2 \mathrm{~cm}$. In a multicenter study of 34 patients treated with 58 ACR procedures, Turner et al reported significant improvements in LL-PI from $29.4^{\circ}$ to $6.6^{\circ}$, LL from $26.7^{\circ}$ to $50.8^{\circ}$, and PT from $28.3^{\circ}$ to $22.1^{\circ}$, but not in T1SPi. ${ }^{9}$ Additionally, they 
concluded that posterior column osteotomies at the ACR level allowed for greater deformity correction, but they did not comment on the sequence of surgical events. Similarly, at final follow-up of 17 patients that underwent ACR, (10 with an AP and 7 with a PAP sequence), we found meaningful improvements in LL, LL-PI, SVA, and T1SPi in both groups, and PT in the AP group. The correction achieved at the ACR level (represented by MSA) was greater with a pre-ACR posterior release (PAP group); however, our study likely lacked the power to detect a statistical difference. The similarity in overall correction in these cohorts may be attributable to a greater number of ACR procedures per patient in the AP group. In fact, the overall MSA change attributable to ACR in the AP group $\left(17.3^{\circ} \mathrm{MSA}\right.$ change per $\mathrm{ACR} \times 1.5 \mathrm{ACR}$ procedures per patient $=25.9^{\circ}$ ) was nearly identical to the PAP group $\left(25.3^{\circ}\right)$. Our complication rate of $29 \%$ was similar to rates in the literature, which range from $14 \%$ to $47 \%{ }^{8}$ Our results indicate that a pre-ACR posterior release may allow for greater correction at a single level. When considering a preACR posterior release, many factors must be considered including the relative stiffness of the segments in question, presence of instrumentation, presence of a vacuum disc phenomenon, and degree of facet hypertrophy. An additional consideration is the rare but life-threatening risk of vascular compromise when the anterior column is lengthened in patients with calcified vessels, which could be a contraindication to the ACR procedure. Importantly, age should be considered when planning a deformity correction, as older patients tolerate a higher degree of sagittal imbalance and overcorrection beyond age-adjusted targets contributes to proximal junctional kyphosis. ${ }^{15}$

Our results should be considered in the context of many limitations. First, because of the retrospective nature, the patients were not matched or randomized and thus, are subject to selection bias and confounding. We attempted to control for this bias by reporting baseline radiographic parameters, the calculated change in radiographic parameters, change in SRS-Schwab classification grade, and degree of correction at the ACR level. However, due to the retrospective nature, we were unable to accurately report on clinically important factors such as endplate damage, subsidence, or degree of facet hypertrophy at the ACR levels. Second, because our sample size, our study may lack the power to detect a difference between the groups (subject to type II error). Third, our primary outcomes were radiographic change rather than clinical improvement. We also were unable to report on cost, readmission rates, or other risk factors for complications such as patient comorbidities or operative time. ${ }^{18,19}$ Fourth, while our final followup time would ideally be longer, our primary objective was to determine if a PAP sequence allowed greater sagittal correction than an AP sequence and given our sample size, we felt our follow-up was adequate and if follow-up was extended, subsequent changes in radiographic parameters could not be attributed to surgical sequence. The limitations listed here are not exhaustive.

In conclusion, we present radiographic outcomes following ACR for rigid sagittal deformity for 2 different technical approaches. Our results suggest that clinically meaningful improvement in sagittal radiographic parameters can be achieved with or without pre-ACR posterior release. To our knowledge, we are the first to show that pre-ACR posterior release may achieve greater correction at the ACR level but is not always necessary to achieve clinically meaningful correction of the sagittal plane deformity. While our study was limited due to patient sample size, our results and limitations should be applied to design future prospective studies to better define specific indications for a pre-ACR posterior release.

\section{REFERENCES}

1. Schwab F, Dubey A, Gamez L, et al. Adult scoliosis: prevalence, SF-36, and nutritional parameters in an elderly volunteer population. Spine (Phila Pa 1976). 2005;30(9):10821085 .

2. Glassman SD, Berven S, Bridwell K, Horton W, Dimar JR. Correlation of radiographic parameters and clinical symptoms in adult scoliosis. Spine (Phila Pa 1976). 2005;30(6):682-688.

3. Glassman SD, Bridwell K, Dimar JR, Horton W, Berven $\mathrm{S}$, Schwab F. The impact of positive sagittal balance in adult spinal deformity. Spine (Phila Pa 1976). 2005;30(18):20242029.

4. Lafage V, Schwab F, Patel A, Hawkinson N, Farcy JP. Pelvic tilt and truncal inclination: two key radiographic parameters in the setting of adults with spinal deformity. Spine (Phila Pa 1976). 2009;34(17):E599-E606.

5. Joseph SA Jr, Moreno AP, Brandoff J, Casden AC, Kuflik P, Neuwirth MG. Sagittal plane deformity in the adult patient. J Am Acad Orthop Surg. 2009;17(6):378-388.

6. O’Neill KR, Lenke LG, Bridwell KH, et al. Clinical and 
radiographic outcomes after 3-column osteotomies with 5-year follow-up. Spine (Phila Pa 1976). 2014;39(5):424-432.

7. Diebo BG, Passias PG, Marascalchi BJ, et al. Primary versus revision surgery in the setting of adult spinal deformity: a nationwide study on 10,912 patients. Spine (Phila Pa 1976). 2015;40(21):1674-1680.

8. Saigal R, Mundis GM Jr, Eastlack R, Uribe JS, Phillips FM, Akbarnia BA. Anterior column realignment (ACR) in adult sagittal deformity correction: technique and review of the literature. Spine (Phila Pa 1976). 2016;41(Suppl 8):S66-S73.

9. Turner JD, Akbarnia BA, Eastlack RK, et al. Radiographic outcomes of anterior column realignment for adult sagittal plane deformity: a multicenter analysis. Eur Spine J. 2015;24(Suppl 3):427-432.

10. Bridwell KH. Decision making regarding Smith-Petersen vs. pedicle subtraction osteotomy vs. vertebral column resection for spinal deformity. Spine (Phila Pa 1976). 2006;31(19 Suppl):S171-S178.

11. Schwab FJ, Blondel B, Bess S, et al. Radiographical spinopelvic parameters and disability in the setting of adult spinal deformity: a prospective multicenter analysis. Spine (Phila Pa 1976). 2013;38(13):E803-E812.

12. Schwab F, Ungar B, Blondel B, et al. Scoliosis Research Society-Schwab adult spinal deformity classification: a validation study. Spine (Phila Pa 1976). 2012;37(12):1077-1082.

13. Pellise F, Vila-Casademunt A, Ferrer M, et al. Impact on health related quality of life of adult spinal deformity (ASD) compared with other chronic conditions. Eur Spine J. 2015;24(1):3-11.

14. Smith JS, Klineberg E, Schwab F, et al. Change in classification grade by the SRS-Schwab Adult Spinal Deformity Classification predicts impact on health-related quality of life measures: prospective analysis of operative and nonoperative treatment. Spine (Phila Pa 1976). 2013;38(19):1663-1671.

15. Lafage R, Schwab F, Glassman S, et al. Age-adjusted alignment goals have the potential to reduce PJK. Spine (Phila Pa 1976). 2017;42(17):1275-1282.

16. Schwab F, Patel A, Ungar B, Farcy JP, Lafage V. Adult spinal deformity-postoperative standing imbalance: how much can you tolerate? An overview of key parameters in assessing alignment and planning corrective surgery. Spine (Phila Pa 1976). 2010;35(25):2224-2231.

17. Murray G, Beckman J, Bach K, Smith DA, Dakwar E, Uribe JS. Complications and neurological deficits following minimally invasive anterior column release for adult spinal deformity: a retrospective study. Eur Spine J. 2015;24(Suppl 3):397-404.

18. Lee NJ, Kothari P, Kim JS, et al. Early complications and outcomes in adult spinal deformity surgery: an NSQIP study based on 5803 patients. Global Spine J. 2017;7(5):432440.

19. Lee NJ, Shin JI, Kothari P, et al. Incidence, impact, and risk factors for 30-day wound complications following elective adult spinal deformity surgery. Global Spine J. 2017;7(5):417424.

Disclosures and COI: IRB Approval 00085402. The author(s) received no financial support for the research, authorship, and/or publication of this article.

Corresponding Author: Byron Stephens, MD, Department of Orthopaedics and Rehabilitation, Vanderbilt University Medical Center, 1215 21st Avenue South, Suite 4200, MCE-South Tower, Nashville, TN 37232. Phone: (913) 221-8212; Email: jeffrey.hills@vumc.org.

Published 30 April 2019

This manuscript is generously published free of charge by ISASS, the International Society for the Advancement of Spine Surgery. Copyright (c) 2019 ISASS. To see more or order reprints or permissions, see http://ijssurgery.com. 